\title{
SLIMs: Smart Lightweight Indoor Maps
}

\author{
Sathittham Sangthong ${ }^{\mathrm{a}}$, Boonchana Purahong ${ }^{\mathrm{b}}$, Attasit Laskul ${ }^{\mathrm{c}}$ \\ Faculty of Engineering \\ King Mongkut's Institute of Technology Ladkrabang \\ Bangkok, Thailand \\ as.sathittham@gmail.com; ${ }^{b}$ yuna001@gmail.com; ${ }^{c}$ klattasi@kmitl.ac.th
}

\begin{abstract}
This paper presents a smart lightweight indoor navigation system called "SLIMs". The system divided into 3 main parts, 1) Indoor Positioning system 2) Indoor Navigation system 3) Administration Management system. SLIMs system takes advantage of the development of short length communication technology or Near Field Communication (NFC) combined with Quick Respond Code (QR Code) with have a very low cost. According to these technologies, it can help to identifying the exact location of the user and facilitated by scanning the tag that is placed all over the building. This system also combined with independent navigation system based on Inertial Navigation System (INS) or Dead Reckoning (DR) method, to give an instruction to the user to reach the destination more accurately. This system also comes with the management of the administrator who can work online. All of this will help in terms of saving cost the devices, less complicated installation, low cost of system maintenance and energy savings as well.
\end{abstract}

Keywords- indoor map; indoor navigation; NFC; QR code; Inertial Navigation System; Dead Reckoning

\section{INTRODUCTION}

The outstanding of computing capabilities, the rapid advances in mobile device and wireless communication combination with the wealth of sensors, such as GPS (Global Positioning System), accelerometer, gyroscope, camera and wireless receivers, are driving the Location Based Services (LBS) application as one of the potential mobile application.

Location service in outdoor have been success the last few years and the next step will be repeating success of location service in outdoor use for navigation and local search in the indoor environment [1]. Outdoor navigation systems are generally based on GPS which is space based global navigation satellite system. It has a good accuracy in open space environment, but unfortunately it does not work well in an indoor environment [2].

Indoor navigation has become a recent research area due to the unavailability of GPS indoor environment. To overcome this problem, variety of technologies and methodologies had been tested and new design had been presented. The existing solutions for indoor navigation systems are generally grouped as network based navigation system and independent navigation system. Network based navigation system such as Bluetooth, Wireless Fidelity (WiFi), Ultra Wide Band (UWB) or Radio Frequency Identification (RFID). Independent navigation system based such as Inertial Navigation System (INS) or Dead Reckoning (DR) method [3]. Position accuracy varies according to the technology and methodology used.

Which several of existing indoor navigation system, there are some factors that we need to consider in order to make indoor navigation system reality, the technology must be low-cost, low-power, have low maintenance expenses and require minimal amount of new infrastructure [1].

This paper presents a smart lightweight indoor navigation system called SLIMs. The system takes advantage of a lowcost wireless technology called Near Field Communication (NFC) and Quick Respond code (QR code) technology. In order to get users current location, users need only to tab smartphone which have NFC capability or camera module and embedded application to reference tag (NFC and QR code). Users can select their desire destination on application and it will always show optimal route to the destination with additional of turn by turn navigation direction while users are moving. SLIMs system also comes with web base administration management system to help admin to manage their indoor map easily.

\section{A. Related Technology}

- NFC

Currently, NFC is one of the enablers for ubiquitous computing. NFC technology can be identified as a combination of contactless identification and interconnection technologies. NFC operates between two devices in a short communication range via a touching paradigm. NFC operates in a frequency band of $13.56 \mathrm{MHz}$ and supports various data transfer rates that reach can up to $424 \mathrm{Kbps}$. It requires touching two NFC-compatible devices together over a few centimeters. NFC communication occurs between an NFC mobile device on one side and an NFC tag (a passive RFID tag), an NFC reader, or an NFC mobile device on the other side [4].

- QR code

QR code is the trademark for a type of matrix barcode (or two-dimensional barcode). A barcode is an optically machine-readable label that is attached to an item and that records information related to that item. The information encoded by a QR code may be made up of four standardized types of data (numeric, alphanumeric, byte/binary, Kanji) or, through supported extensions, virtually any type of data [5].

- Inertial Navigation System 
An INS is a navigation aid that uses a computer, motion sensors (accelerometers) and rotation sensors (gyroscopes) to continuously calculate via dead reckoning the position, orientation and velocity (direction and speed of movement) of a moving object [6].

\section{B. Related work}

The indoor navigation systems that author [3] have designed that use NFC technology to locate the current user location inside the building. The system only provide the shortest path feature to the selected destination and it only support on smartphone that have NFC capability. On the indoor navigation system of author [2], the system also use the same indoor positioning technique (NFC technology) but there are more extra features like place information, search, car parking, feedback, survey, nearest toilet, etc. and able to use with others smartphone that have capability of camera to scan QR code as the same as NFC tag. On the other hand, SLIMs system provides more information to user on navigation system by give turn by turn direction navigation. The proposed system also provides new feature as web base administration system in order to make the system less complicated in term of installation and maintenance.

With other indoor navigation systems, there are several of technologies and methodologies. Wifarer is an indoor navigation system that offers a set of maps for positioning indoors. The system uses $\mathrm{Wi}-\mathrm{Fi}$ fingerprinting technique with an accuracy of about $1.3 \mathrm{~m}$, uses an individual Maps. The system is designed for iOS and Android [7]. Compare with SLIMs system, Wifarer need to add Wi-Fi access point as new infrastructure. In term of cost and complication of implementation, SLIMs is less cost expense and less complicated. Micello is another indoor map guidance application for Android and iOS. The system uses GPS to find user location outdoor and display on custom google map [8]. In order to use navigation user need to manual selected the start point and the destination from the map. However SLIMs system utilizes NFC technology and QR code to facilitate the process of finding user current location.

\section{SySTEM ARCHITECTURE AND DESIGN}

\section{A. System Architecture}

The overview of system architecture of SLIMs is show in Fig. 1, the SLIMs system is designed to be simple and user friendly. The user only needs to have a smartphone that have NFC feature or camera module. Then the user needs to simple tab or scan reference tag. In case of user don't have SLIMs application, it will ask the user to download application from internet. In case of user already have SLIMs application; it will automatically start an application and show map information. The user can selects destination inside the building, SLIMs application will show optimal route to the selected destination. As user starts walking to the destination, SLIMs application will show instructions to user and estimate how far to reach the destination. During walking to the destination, user can tab or scan reference tag to fix user's current position on the map.

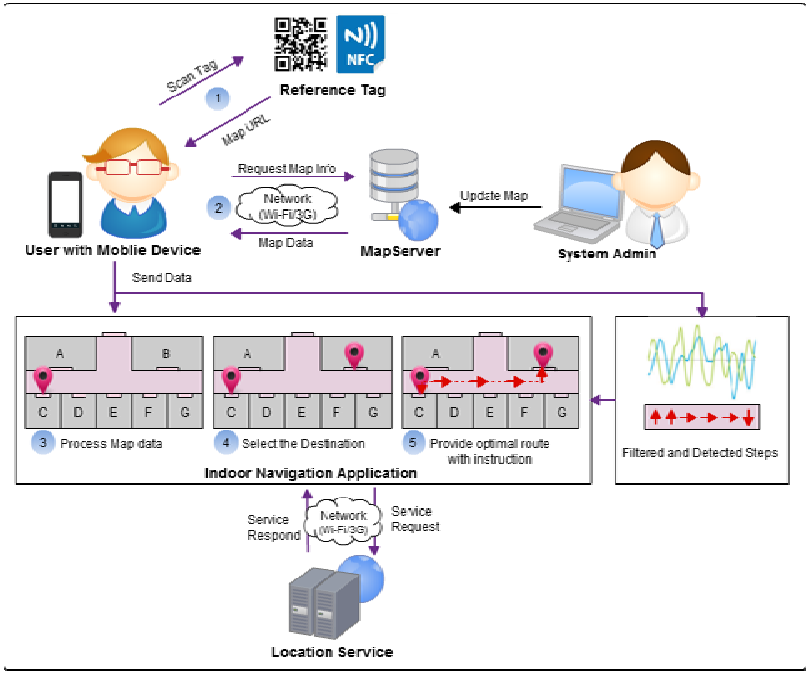

Figure 1. SLIMs system architecture

\section{B. System Design}

The system design had been divided into 3 main parts, 1) Indoor positioning system 2) Indoor navigation system 3) Administration management system

1) Indoor Positioning System. The capability to determine a user's positioning within a building is a necessary part of a navigation system. In order to get user's current position, the SLIMs system uses reference tags that spread over the building as shown in Fig. 2, which this solution of positioning, we can save cost in term of system infrastructures, system installation and system maintenance compare to other solutions and still friendly with the user.

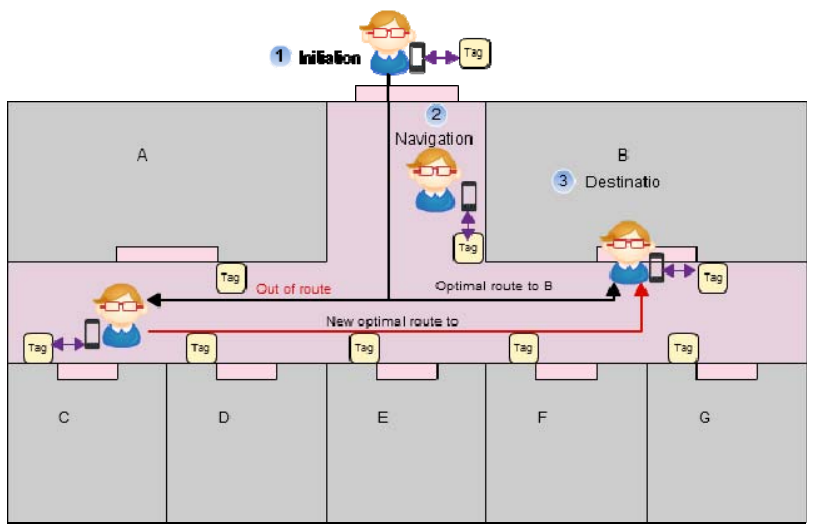

Figure 2. Indoor Positioning and Navigation

2) Indoor navigation system. The navigation system is responsible for determining an optimal route to a destination. It consists of graphing functionality and routing algorithm.

- Graphing

With the current position of the user known, it is important to determine the destination that user wants to go to. The output of this module is a path that guides the user to 
their desired destination. In order to achieve this task, we choose to represent the map of the building in a graph on which the routing algorithm can operate. Fig. 3 shows the example of graph in the indoor environment.

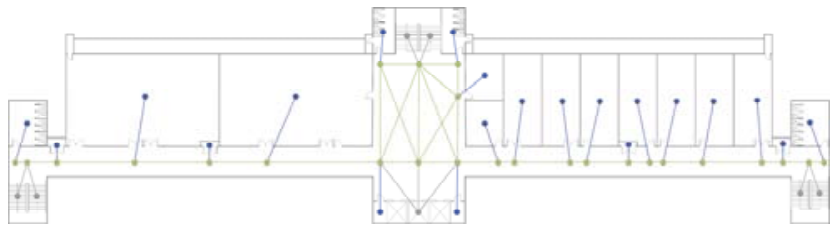

Figure 3. The graph of example building

- Inertial Navigation system

To offer continuous navigation experience, the SLIMs system using INS base on Pedestrian Dead Reckoning (PDR) algorithm to detect steps and to estimate each step's length according to the user current movement. Fig. 4 shows the basic concept of turn by turn direction navigation system of SLIMs.

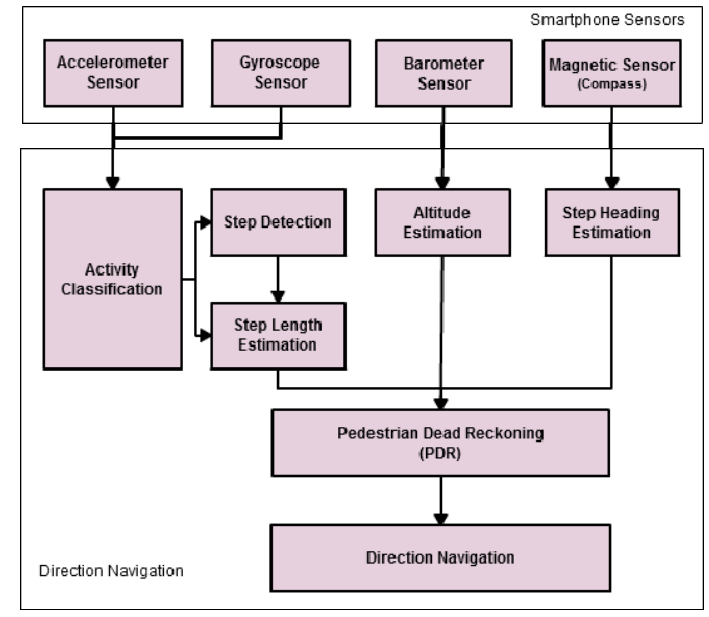

Figure 4. Basic overview of direction navigation

- $\quad$ Routing Algorithm

We chose Dijkstra's algorithm to find the shortest path between two nodes in a graph system for SLIMs's routing algorithm. Dijkstra's algorithm always provide shortest path from single source to one or multiple destinations and the complexity of Dijkstra when well implemented is $\mathrm{O}(\mathrm{NlogN})$ which suitable for indoor environment with a moderate number of nodes.

3) Administration Management System. In order to make an indoor navigation system in reality, it isn't only about the cost of the system but also about the complexity of installation and cost of system maintenance. So we introduce to our web base administration management system to help system admin and to overcome these problems. SLIMs's administration management system lets user create their own indoor map overlay on Google maps engine by using the building floor plan. The user can create their reference tag from this system as well.

\section{PROTOTYPE IMPLEMENTATION}

For this implementation is to test the design of SLIMs system within university campus. The software application of SLIMs is implemented on the Google Android phone, the Samsung Galaxy Note 2. The user interface of SLIMs application and the basic features such as get user current location, direction to selected destination or search place is show in Fig. 5, the system consists of 3 parts which are positioning system with android application, navigation system and administration management system.
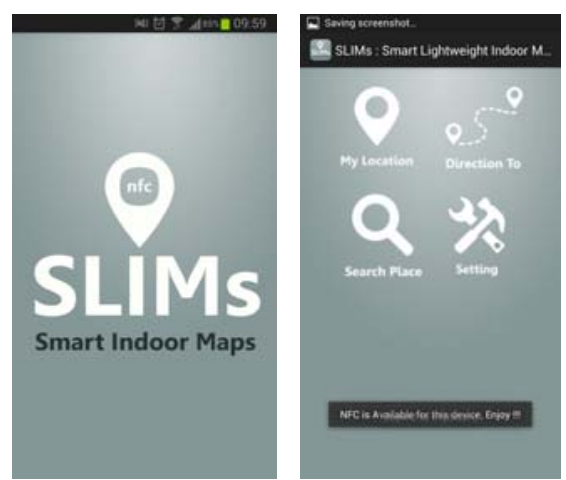

Figure 5. SLIMs application and features

Fig. 6 shows the reference tag that we use to get user's current position in indoor map, it's designed to be both NFC tag and QR code. NFC tag in this implementation is Mifare $1 \mathrm{~K}$ tag. The system admin also be able to write NFC tag by smartphone and create QR code from the administration management system as well.

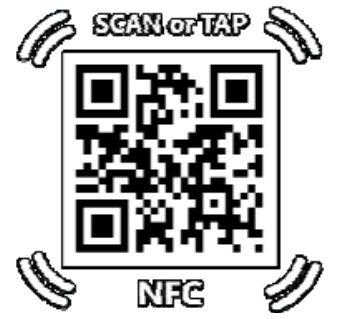

Figure 6. Reference Tag with NFC tag and QR code

When user enters to the building for the first time, he/she need to scan one of reference tag in order to download application or the information related to the building. The indoor map will be downloading to user phone from MapServer via OTA (Over the Air). After that, user will now know his/her current position and be able to navigate to any places inside this building as shown in Fig. 7, user can searches anywhere inside the building and the system will always show the optimal route to the destination, while searching user is able to see the detail for more information of that place as well. 

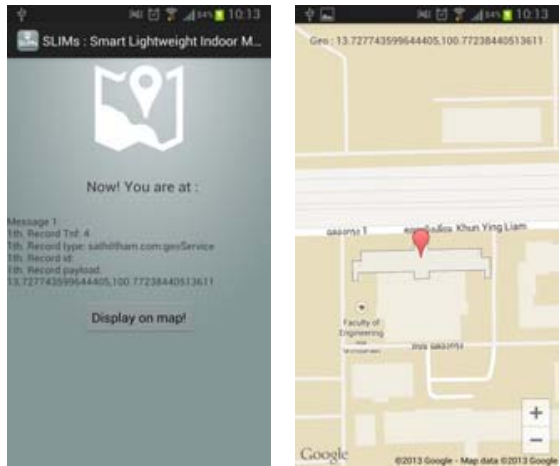

Figure 7. Get user's current location by scanning reference tag

The inertial navigation system use sensors of Samsung Galaxy Note 2, including the accelerometer, gyroscope and digital compass (magnetic field sensor). The sensors need to be calibrated to correct errors before the data is processed. The accelerometer and gyroscope chip, build-in the Galaxy Note 2 is LSM330DLC and the magnetic field sensor is Asahi Kasei's AK8963C. Fig. 8 shows turn by turn direction navigation will be shown as user starts navigation along the map.
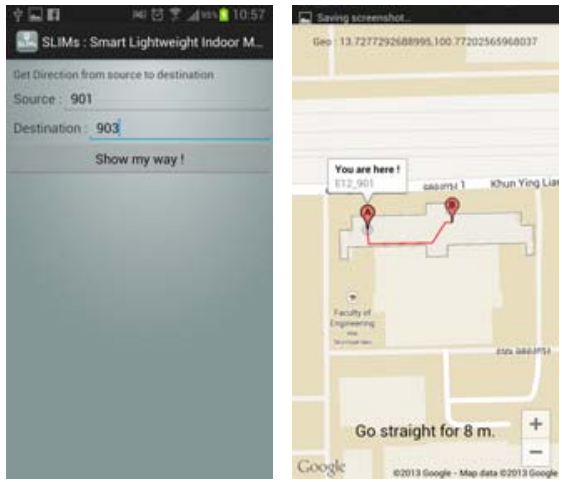

Figure 8. Navagation with the optimal route

The web based administration management system is of another support system as shown in Fig.9, it's implemented with PHP and MySQL database. The admin need to be verified by login system to be able to create maps, edit, update, delete data.

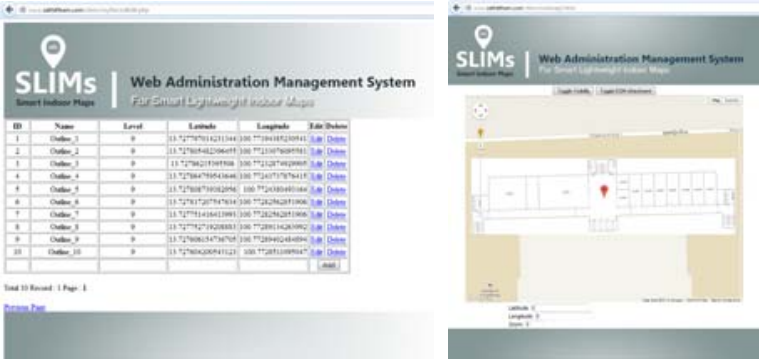

Figure 9. Web Administration Management System

\section{CONCLUSION}

This paper had detailed in indoor navigation system design and implemented in the university campus. The android smartphone that have ability of NFC and camera module can use the application to scan the reference tag to identify the user current position in the building. The map can show the current position of the user and display the detail of the indoor map inside the building. User can select the destination to go and the system will always show the optimal route. This presented system will help visitor in the public building such as shopping mall, hospital, museum, or exhibition to reach their destination easily with turn by turn direction navigation system. This system also saves the cost of device, easy to implement, low maintenance expense and low power.

\section{REFERENCES}

[1] Lauri Wirola, Tommi A. Laine and Jari Syrjarinne, "Mass-market requirement for indoor posistioning and indoor navigation." 2010 International Conference on Indoor Posisioning and Indoor Navigation (IPIN), 15-17 september 2010.

[2] Omran Al Hammadi, Ahmed Al Hebsi, M. Jamal Zemerly, "Indoor localization and guidance using portable smartphone,” 2012 IEEE/WIC/ACM International Conferences on Web Intelligence and Intelligent Agent Technology.

[3] Busra Ozdenizci, Kerem Ok, Vedat Coskun, Mehmet N. Ayadin, "Development of an indoor navigation system using NFC technology,” 2011 Fourth International Conference on Information and Computing.

[4] Vedat Coskun, Kerem Ok, Busra Ozdenizci, "Professional NFC application development for android.” Wrox. pp. 5-22, April 2013.

[5] Wikipedia,QR code, Available: http://en.wikipedia.org/wiki/QR code Access date : 23 August 2013.

[6] Wkipedia, Inertail navigation system, Available: http://en.wikipedia.org/wiki/Inertial_navigation_system, Access date : 23 August 2013.

[7] Wifarer, Indor positioning|indoor GPS|location analysis, Available : htpp://www.wifarer.com, Access date: 23 August 2013.

[8] Micello, Available: http://www.micello.com, Access date: 23 August 2013 